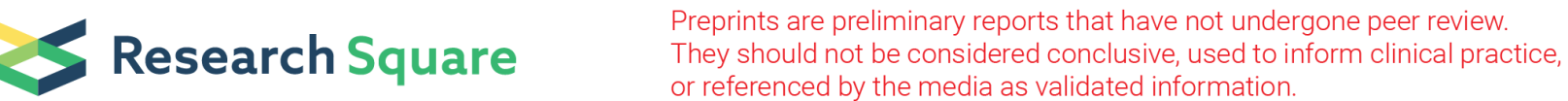

\section{A preliminary study of quantitative analysis of tissue perfusion using contrast-enhanced ultrasound in the evaluation of the severity of acute pancreatitis}

\section{Liang Li}

Tianjin Nankai Hospital https://orcid.org/0000-0001-9086-7422

\section{$\mathrm{Xin} \mathrm{Xu}$}

Tianjin Nankai Hospital

\section{Guangxia Wang}

Tianjin Nankai Hospital

Yunfeng Cui ( $\nabla$ dr_cui001@163.com )

\section{Research article}

Keywords: Acute pancreatitis, Contrast enhanced ultrasound, Tissue perfusion

Posted Date: June 1st, 2020

DOI: https://doi.org/10.21203/rs.3.rs-31531/v1

License: (c) (i) This work is licensed under a Creative Commons Attribution 4.0 International License.

Read Full License 


\section{Abstract \\ Background}

Acute pancreatitis (AP) is a lethal disease with high mortality. Early diagnosis and evaluation of the severity of pancreatitis is crucial for the prevention a treatment of AP. The clinical value of contrastenhanced ultrasound (CEUS) in evaluating the severity of acute pancreatitis by quantitative analysis of the tissue perfusion and the areas of pancreatic parenchymal necrosis is little known.

\section{Methods}

60 acute pancreatitis patients (AP group) and 30 volunteer (control group) were selected, the AP group was further divided into subgroup, the mild acute pancreatitis (MAP) group and the severe acute pancreatitis (SAP) group. All received CEUS examination, the differences of the parameters of timeintensity curve (TIC) between group AP and Control were analyzed. The receiver operating characteristics (ROC) curve of CEUS in diagnosing AP and SAP were drew, then calculated the best cut-off point of CEUS for the diagnosis of AP.

\section{Results}

Compared to group Control, there was a marked increase in AT and TTP, while a dramatic decrease in PI and AUC in group AP (all $P<0.05$ ), no significant difference of WT and $S$ was observed. After analysis of $\mathrm{PI}$ and AUC of those two subgroup AP patients, it was much higher in MAP group than SAP group, in contrast, the TTP decreased markedly(all $P<0.05$ ), no significant difference of AT,WT and $S$ was found. Next, we found that the sensitivity, specificity and diagnostic coincidence rate were $96.7 \%, 73.3 \%$ and $85.0 \%$ respectively, when the AUC $\leq 727.2 \mathrm{dBs}$ was used as the standard for the diagnosis of AP; and the sensitivity, specificity and diagnostic coincidence rate were $93.3 \%, 73.3 \%$, and $83.3 \%$, respectively when $\mathrm{PI} \leq 11.41 \mathrm{~dB}$ was used as the standard for the diagnosis of SAP. Finally, we found that there was no significant difference in the detection of pancreatic parenchymal necrosis between CEUS and contrastenhanced computed tomography (CECT).

\section{Conclusions}

CEUS could provide an objective and powerful basis for the early diagnosis of SAP, and also detect necrotic areas of pancreatic parenchyma early, quickly and accurately, which provide an available tool for clinic to early detection and assessment of the severity of acute pancreatitis.

\section{Background}


Acute pancreatitis (AP) is a disease with potentially lethal out-come ${ }^{[1]}$. AP can be divided into mild acute pancreatitis (MAP) and severe acute pancreatitis (SAP), which is an inflammatory disorder of the pancreas that has been clinically defined as a common form of acute abdominal pain ${ }^{[2-4]}$.MAP has a good prognosis and few complications with low mortality. However, SAP accompanied by serious complications is reported to have a high mortality rate ${ }^{[5,6]}$. Although SAP accounts for only $15-25 \%$ of AP cases, its mortality rate may be as high as $15-48.4 \%{ }^{[7]}$. Early diagnosis and evaluation of the severity of pancreatitis is crucial in AP management. As a continuous injury mechanism, pancreatic microcirculation disorder runs through the whole process of pancreatitis development and is an important factor in the transformation of hemorrhage and necrosis. Therefore, early detection of pancreatic microcirculation changes is the key to evaluate the severity of pancreatitis ${ }^{[8,9]}$.

Contrast enhanced ultrasound (CEUS) is a new technique which can display imperceptible blood-vessel and tissue perfusion of microcirculation, and can also provide information on the vascularization of the pancreatic parenchyma and can differentiate between areas of inflammation and areas of necrosis ${ }^{[10]}$.

CEUS takes advantage of its special features: the high contrast and spatial resolution, the use of a bloodpool microbubble contrast agent and the real-time, dynamic evaluation of tissue enhancement, filtering the background tissue signals ${ }^{[11]}$. The introduction of CEUS has led to major improvements in the diagnostic capabilities of ultrasound (US) ${ }^{[12]}$. CEUS is less expensive compared to computed tomography(CT) and magnetic resonance imaging(MRI) and is able to significantly improve the accuracy of US, allowing better characterization and staging of pancreatic pathologies ${ }^{[13]}$.

CEUS may improve the detection and delimitation of the necrotic areas, which appear completely a vascular ${ }^{[14]}$. Unfortunately, in the literature there are no studies comparing CEUS with CT in the quantitative evaluation and follow-up of AP. At this moment, CT remains more effective than CEUS, in particular in grading the stage of the disease.

The prospective study included 60 patients with pancreatitis, all of whom were performed CEUS examination and applies the time-intensity curve (TIC) quantitative analysis. The purpose is to explore the perfusion characteristics of pancreatic tissue by CEUS, evaluating the clinical value of CEUS in AP diagnosis, which achieves early identification of SAP, evaluating its severity and providing reliable imaging basis for clinical treatment and efficacy evaluation.

\section{Methods}

A randomized controlled trial was conducted to compare the CEUS parameters of AP, thus determining the best cut-off point between AP and SAP, and evaluating the diagnostic value of CEUS.

\section{Patient data}


This study was a prospective study. It was conducted in accordance with the Declaration of Helsinki, and with approval from the Ethics Committee of Tianjin Nankai Hospital (Tianjin, China). Written informed consent was obtained from all participants. In total, 60 inpatients given a clinical diagnosis of AP (30 patients with MAP and 30 patients with SAP) at Tianjin Nankai Hospital from May 2014 to September 2016 were selected for this study. All patients were taken the examination by CEUS.

\section{Inclusion criteria}

The inclusion criteria for cases of AP were mainly based on the Chinese guidelines for diagnosis and treatment of $A P^{[15]}$.

MAP group: acute persistent abdominal pain (a few had no abdominal pain), serum amylase activity increased (few without increase), no local complications (pancreatic necrosis, pseudocyst, pancreatic abscess). No organ failure; Ranson score < 3; APACHE 『score < 8; Balthazar CT grading below grade Il; Chinese CT classification is A, B, C.

SAP group: acute persistent abdominal pain (a few had no abdominal pain), serum amylase activity increased $\geq 3$ times the upper limit of normal value, and patients with one of the following: local complications (pancreatic necrosis, pseudocyst, pancreatic abscess); Organ failure; Ranson score $\geq 3$; APACHE $\llbracket$ score $\geq 8$; Balthazar CT grade囚or above; Chinese CT classification was D and E.

\section{Exclusion criteria}

After laboratory examination and imaging examination, patients were clinically diagnosed with chronic pancreatitis, or patients with acute onset of chronic pancreatitis and pancreatic cancer.

\section{CEUS examination}

The PHILIPS iU22 (Philips Medical Systems, Bothell, WA, USA) ultrasonographic system with a corresponding $\mathrm{C} 5-1-\mathrm{MHz}$ transducer was used. The ultrasonographic system was quipped with harmonic contrast pulse sequencing technology. The contrast agent used was SonoVue (Bracco SpA, Milan, Italy), which is a suspension of stabilized sulfur hexafluoride microbubbles.

The CEUS program was at a low mechanical index (MI), MI was set at 0.05 . A $1.2-1.5 \mathrm{ml}$ volume of contrast agent suspension (SonoVue) was administered as a bolus injection through the median cubital vein, followed by flushing with $5 \mathrm{ml}$ saline solution. After an $8-15 \mathrm{sec}$ delay, the pancreatic parenchyma began to undergo enhancement. The real-time contrast-enhanced image obtained after contrast agent injection was recorded on a hard disk, and the injection times were calculated simultaneously ${ }^{[16]}$. The CEUS image data were analyzed with QLAB 10 advanced ultrasound quantification software, and the TIC was obtained.

The TIC parameters include: 1. Arise time (AT): time to begin enhancement. 2. Time to peak (TTP): the time to reach the peak. 3. Wash-time (WT): the total withdrawal of the contrast agent from the pancreatic parenchyma. 4. Peak intensity (PI): the perfusion volume at the peak time. 5. The slope of the ascending 
branch of the curve (sharpness, S): it is related to the rapid degree of tissue perfusion and clearance. 6 . Area under the curve (AUC): it is proportional to the average blood flow in the area of interest.

\section{Statistical analysis}

SPSS 22.0 and Graph Pad Prism8 software were used for statistical analysis of the data. Measurement data conforming to normal distribution were expressed as mean \pm standard deviation $(M \pm S D)$, and measurement data not conforming to normal distribution were expressed as median (M). Two independent samples t-test and paired t-test were used as statistical methods. For all analyses, values of $P<0.05$ were considered statistically significant. Drawing the receiver operating characteristics (ROC) curves for statistically significant parameters; The best cut-off point of AP and SAP was found according to the highest critical point of the Youden index, and the sensitivity, specificity and coincidence rate were calculated ${ }^{[17]}$.

\section{Results}

\section{General characteristics}

30 patients in the AP group including 43 males and 17 females, aged 24-71 years old, with an average age of $(49.5 \pm 11.7)$ years old. 30 patients in the control group, including 17 males and 13 females, aged from 32 to 77 years old, with an average age of $(52.5 \pm 10)$ years old. Among the 60 AP patients, 22 $(36.7 \%)$ had biliary pancreatitis, 17 (28.3\%) had alcoholic pancreatitis, 5 (8.3\%) had binge-eating pancreatitis, $15(25 \%)$ had hyperlipidemia pancreatitis, and $1(1.7 \%)$ had unknown cause. Among the 30 cases of MAP, only 1 case developed secondary pulmonary infection, while 2 cases developed SAP secondary heart failure, 4 cases developed acute respiratory distress syndrome (ARDS), 3 cases developed pulmonary infection, 3 cases developed shock and 5 cases developed renal insufficiency (Table 1).

Table 1

Comparison of clinical outcomes (complications) between MAP and SAP(n/\%)

\begin{tabular}{|llllll|}
\hline Group & Pulmonary infection & Heart failure & ARDS & Shock & Renal insufficiency \\
\hline $\operatorname{MAP}(n=30)$ & $1(3.3)$ & $0(0)$ & $0(0)$ & $0(0)$ & $0(0)$ \\
$\operatorname{SAP}(n=30)$ & $3(10)$ & $2(6.7)$ & $4(13.3)$ & $3(10)$ & $5(16.7)$ \\
\hline
\end{tabular}

\section{Quantification of pancreatic perfusion in AP patients by time-intensity curves}

After analyzing the TIC parameters, it indicated that AT and TTP in AP group were much higher than those in control group ( $9.64 \pm 1.50$ vs $8.57 \pm 0.96 \mathrm{~s}, 16.21 \pm 3.47$ vs $12.92 \pm 3.51 \mathrm{~s}$, all $\mathrm{P}<0.05)$, in contrast, the value of $\mathrm{PI}$ and AUC in AP group decreased significantly compared to those in control group (10.90 \pm 2.82 vs $16.19 \pm 3.85 \mathrm{~dB}, 560.73 \pm 190.97$ vs $867.39 \pm 243.53 \mathrm{dBs}$, (all $\mathrm{P}<0.05)$, which make it available to 
identify the AP patients, there was no significant difference of WT and S between the AP group and control group (Table 2, Fig. 1, Fig. 3).

Table 2

Comparison of TIC parameters between AP group and control group ( $\mathrm{M} \pm$ SD)

\begin{tabular}{|lllll|}
\hline Parameters & AP $(\boldsymbol{n}=\mathbf{6 0})$ & Control $(\boldsymbol{n}=\mathbf{3 0})$ & $\boldsymbol{t}$ & $\boldsymbol{P}$ \\
\hline AT(s) & $9.94 \pm 1.87$ & $8.57 \pm 0.96$ & 3.761 & $<0.001$ \\
\hline TTP(s) & $16.21 \pm 3.47$ & $12.92 \pm 3.51$ & 4.221 & $<0.001$ \\
\hline WT(s) & $46.16 \pm 6.34$ & $49.27 \pm 7.38$ & -1.92 & 0.064 \\
\hline PI(dB) & $10.90 \pm 2.82$ & $16.19 \pm 3.85$ & -7.406 & $<0.001$ \\
\hline S & $1.57 \pm 0.78$ & $1.44 \pm 0.71$ & 0.640 & 0.528 \\
\hline AUC(dBs) & $560.73 \pm 190.97$ & $867.39 \pm 243.53$ & -6.538 & $<0.001$ \\
\hline
\end{tabular}

\section{Quantification of pancreatic perfusion in the subgroup of AP patients by time-intensity curves}

Next, to determine whether the CEUS could grade the stage of the AP patients, we evaluate the value of TIC between the AP patients, who were divided into the MAP and SAP based on the clinical guideline. We found that TTP increased dramatically in SAP patients (17.52 \pm 2.52 vs $14.90 \pm 3.82 \mathrm{~s}, P<0.05)$, both PI and AUC in SAP group decreased significantly $(9.40 \pm 2.54 \mathrm{vs} 12.40 \pm 2.26 \mathrm{~dB}$, and $453.81 \pm 141.54 \mathrm{vs}$ $667.64 \pm 174.69 \mathrm{dBs}$, respectively, all $P<0.05)$. No significant difference of $A T, W T$ and $S$ was found between the SAP group and MAP group (Table 3, Fig. 2, Fig. 4).

Table 3

Comparison of TIC parameters between SAP group and MAP group (M \pm $\mathrm{SD})$

\begin{tabular}{|lllll|}
\hline Parameters & MAP $(\boldsymbol{n}=30)$ & $\operatorname{SAP}(\boldsymbol{n}=30)$ & $\boldsymbol{t}$ & $\boldsymbol{P}$ \\
\hline AT(s) & $9.64 \pm 1.50$ & $10.24 \pm 2.18$ & -1.245 & 0.218 \\
\hline TTP(s) & $14.90 \pm 3.82$ & $17.52 \pm 2.52$ & -3.128 & 0.003 \\
\hline WT(s) & $47.22 \pm 5.37$ & $48.36 \pm 6.39$ & -1.82 & 0.163 \\
\hline PI(dB) & $12.40 \pm 2.26$ & $9.40 \pm 2.54$ & 4.831 & $<0.001$ \\
\hline S & $1.61 \pm 0.68$ & $1.55 \pm 0.78$ & -1.126 & 0.637 \\
\hline AUC(dBs) & $667.64 \pm 174.69$ & $453.81 \pm 141.54$ & 5.209 & $<0.001$ \\
\hline
\end{tabular}

The diagnostic value of quantitative parameters of CEUS TIC in AP and SAP 
After calculating the area under the ROC curve of the all TIC parameters, we found that AUC was the most powerful parameter for the diagnosis of $A P$, the area under the ROC curve was $0.87(95 \% \mathrm{Cl}$ : $0.802-0.943)$. The best cut-off point was $727.2 \mathrm{dBs}$ according to the Youden index. When choosing AUC $\leq 727.2 \mathrm{dBs}$ as the standard for the diagnosis of AP, the sensitivity, specificity and diagnostic coincidence rate were $96.7 \%, 73.3 \%$ and $85.0 \%$, respectively(Table 4 , Fig. 3).

Table 4

Diagnostic value of different parameters for AP (\%)

\begin{tabular}{|lllllll|}
\hline Parameters & Sensitivity & $95 \% \mathrm{Cl}$ & Specificity & $95 \% \mathrm{Cl}$ & $\begin{array}{l}\text { Coincidence } \\
\text { rate }\end{array}$ & $\begin{array}{l}\text { Youden } \\
\text { index }\end{array}$ \\
\hline AT $\geq 9.28 \mathrm{~s}$ & 65.0 & $\begin{array}{l}52.4- \\
75.8\end{array}$ & 80.0 & $\begin{array}{l}62.7- \\
90.5\end{array}$ & 72.5 & 0.450 \\
\hline $\mathrm{TP} \geq 14.61 \mathrm{~s}$ & 71.7 & $\begin{array}{l}59.2- \\
81.5\end{array}$ & 83.3 & $\begin{array}{l}66.4- \\
92.7\end{array}$ & 78.9 & 0.550 \\
$\mathrm{PI} \leq 15.34 \mathrm{~dB}$ & 83.3 & $72.0-90.7$ & 80.0 & $\begin{array}{l}62.7- \\
90.5\end{array}$ & 81.7 & 0.633 \\
& & & & & & \\
AUC $\leq$ & 96.7 & $\begin{array}{l}88.6- \\
727.2 \mathrm{dBs}\end{array}$ & 73.3 & $\begin{array}{l}55.6- \\
85.8\end{array}$ & 85.0 & 0.700 \\
\hline
\end{tabular}

Next, we found that PI was the most powerful parameter to grade the stage of the AP patients by CEUS, the area under the ROC curve was $0.85(95 \% \mathrm{Cl}$ : 0.754-0.952). The best cut-off point was $11.41 \mathrm{~dB}$ according to the Youden index. When selecting $\mathrm{PI} \leq 11.41 \mathrm{~dB}$ as the standard for differentiating SAP from MAP, the sensitivity, specificity and diagnostic coincidence rate were $93.3 \%, 73.3 \%$, and $83.3 \%$, respectively(Table 5, Fig. 4).

Table 5

Diagnostic value of different parameters for SAP (\%)

\begin{tabular}{|lllllll|}
\hline Parameters & Sensitivity & $\mathbf{9 5 \%} \mathrm{Cl}$ & Specificity & $\mathbf{9 5 \% \mathrm { Cl }}$ & $\begin{array}{l}\text { Coincidence } \\
\text { rate }\end{array}$ & $\begin{array}{l}\text { Youden } \\
\text { index }\end{array}$ \\
\hline $\mathrm{TP} \geq 14.58 \mathrm{~s}$ & 100 & $\begin{array}{l}88.7- \\
100\end{array}$ & 56.7 & $\begin{array}{l}39.2- \\
72.6\end{array}$ & 78.3 & 0.567 \\
\hline $\mathrm{PI} \leq 11.41 \mathrm{~dB}$ & 93.3 & $\begin{array}{l}78.7- \\
98.8\end{array}$ & 73.3 & $\begin{array}{l}55.6- \\
85.8\end{array}$ & 83.3 & 0.667 \\
$\begin{array}{l}\text { AUC } \leq \\
481.1 \mathrm{dBs}\end{array}$ & 70.0 & $\begin{array}{l}52.1- \\
83.3\end{array}$ & 86.7 & $\begin{array}{l}70.3- \\
94.7\end{array}$ & 78.4 & 0.567 \\
\hline
\end{tabular}

\section{Comparison of the detection rate of peripancreatic local complications between CEUS and CECT}

It is well known that CECT is the imaging modality of choice for diagnosis, staging, and detection of complications of acute pancreatitis, particularly the identification and quantification of peripancreatic necrosis. In this section, we compared the detection rate of peripancreatic local complications between 
CEUS and CECT. And it showed that of 30 SAP patients, 30 cases of pancreatic necrosis, 3 cases of pseudocyst and 7 cases of peripancreatic abscess were diagnosed by CECT, all of which were also diagnosed by CEUS, and the diagnostic coincidence rate was $100 \%$, indicating CEUS could be considered as an alternative tools for the detection of initiation and extension of the peripancreatic necrosis.

(Table 6)

Table 6

Comparison of the detection rate of peripancreatic local complications between CEUS and CECT $(n=30)$

\begin{tabular}{|llll|}
\hline Peripancreatic complications & CECT & CEUS & Coincidence rate(\%) \\
\hline pancreatic necrosis & 30 & 30 & 100 \\
\hline pancreatic pseudocyst & 3 & 3 & 100 \\
\hline peripancreatic abscess & 7 & 7 & 100 \\
\hline
\end{tabular}

\section{Discussion}

For the method to evaluate the severity of acute pancreatitis in the past there are also many, commonly used have Ranson, Glasgow, APACHE $\nabla$ etc., but there are some difficulties in the actual clinical applications ${ }^{[18]}$.CECT examination has good specificity and sensitivity for the evaluation of acute pancreatitis, but there are some problems such as timing and cost. Therefore, it is still necessary to find a convenient, non-invasive, sensitive and specific early evaluation method ${ }^{[19]}$. With the development of ultrasonography and the constant update of ultrasound examination equipment, the rate of diagnosis and diagnosis of acute pancreatitis by US has reached a high level, and it is harmless, economical, convenient and easy to be accepted by patients, so it has become a routine auxiliary diagnosis and dynamic monitoring method for acute pancreatitis ${ }^{[20,21]}$.

CEUS provides a dynamic view of the "vascular pattern" through the exclusively intravascular flow of the microbubbles, assessing the aspect of increased echogenicity through nonlinear signals from the microbubbles ${ }^{[22]}$. The contrast agent is removed from the body by breathing through the lungs, so it can be used in patients with SAP associated renal failure. Unlike the contrast agent used in ultrasonography, the contrast agent used in CT and MRI has a short intravascular retention time, being rapidly cleared from the circulation and reaching the extracellular space, and then excreted by the kidneys ${ }^{[23]}$.

Vitali $\mathrm{F}$ et al. considered that the performance of CEUS in diagnosing SAP to be very good compared to CT (89\% specificity, $95 \%$ sensitivity) ${ }^{[24]}$. Our study compared the detection rate of the areas of pancreatic necrosis in SAP through CECT (gold standard diagnostic) and CEUS, and there was no statistically significant difference in diagnose accordance rate.

Analyzing the current data from literature regarding the use of CEUS in acute pancreatitis, most of them are qualitative. Till now, there are few published studies on the quantitative analysis of pancreatic tissue 
perfusion and determination of diagnostic thresholds for AP and SAP $[25,26]$.

TIC analysis of grey scale signal changes after contrast agent injection is one of the current methods for quantitative ultrasonic study of blood perfusion ${ }^{[27]}$. Among the quantitative parameters, PI has a good correlation with tissue perfusion amount, which refers to the most significant enhancement intensity of contrast agent on the local section, reflecting the maximum dose of contrast agent reaching this section per unit time. AUC is related to the distribution volume, blood flow velocity and perfusion time of the contrast agent. When the dose and instrument conditions are controlled at a constant rate, the AUC is affected by the flow rate and distribution volume, and is linearly related to the blood flow of the substance. In other words, AUC reflects the change of blood flow volume in the blood vessel, which is one of the most valuable parameters in TIC. The PPT is the time from the beginning of the contrast agent entering the tissue to the maximum strength of the enhancement. The change of the upward slope of the curve can reflect the perfusion rate of the contrast agent entering the tissue ${ }^{[28]}$.

The results of TIC analysis in our study showed that TIC in control group was characterized by a sharp arc curve with a steep ascending branch. After reaching the peak, the descending branch decreased gently compared with the ascending branch. In the AP group, TIC presented a relatively flat arc curve, with the ascending branch slowly rising and the descending branch even more slowly extending. Compared with control group, the AT, TTP and WT in AP group were higher than that in control group. The PI and AUC of the contrast agent in AP group were smaller than those in control group, because the small blood vessels and microcirculation were damaged, the blood flow was slow, the perfusion volume was reduced, and the blood flow volume in the blood vessels was reduced. And by analyzing the TIC parameters, we can draw ROC curves for statistically significant parameters. Then we can determine the best cut-off point of AP and SAP.

A limitation of this study is the relatively small number of patients and the diversity in the ultrasonographically assessed structural changes. Our study provides a more objective, rapid, and accurate diagnostic method for the diagnosis of AP and SAP. Together with other clinical and biohumoral information, these ultrasonographic parameters and their statistical analysis can assist in early clinical evaluation of the severity of AP.

\section{Conclusions}

An important advantage of CEUS is the possible real-time assessment of the vascular pattern with higher temporal resolution than other imaging techniques. Besides, "SonoVue" administration can be repeated due to a better patient tolerance and a reduced incidence of adverse reactions.

Our study attests the usefulness of CEUS in detecting the area of necrosis in AP, with similar results to those of CECT. We determined the best cut-off point of AP and SAP. Thus, our study provides a more objective, rapid, and accurate diagnostic method for the diagnosis of AP and SAP, which can assist in 
early clinical evaluation of the severity of acute pancreatitis. And CEUS is a useful tool in the emergency diagnosis and monitoring of severe acute pancreatitis.

\section{Declarations}

\section{Availability of data and materials}

The dataset analyzed during the current study is available from the corresponding author on a reasonable request.

\section{Ethics approval and consent to participate}

This study was carried out under the approval of the Ethics Committee of Tianjin Nankai Hospital. Informed consent was obtained from all patients prior to participation in the study.

\section{Consent for publication}

Not applicable.

\section{Competing interests}

All authors declare that they have no competing interests.

\section{Funding}

This work was supported by the Key Project of Health Industry in Tianjin(16KG159).

\section{Authors' contributions}

Liang Li wrote the first draft and revision of the manuscript. Xin Xu contributed in writing CT image description part. Guangxia Wang contributed in revision of the manuscript and discussion of results. Yunfeng Cui contributed in study design, discussion of results, and approval of the final version. All authors read and approved the final manuscript.

\section{Acknowledgements}

We thank all the participants who participated in this study. 


\section{References}

1. Kitterer D, Artunc F, Segerer S, Alscher MD, Braun N, Latus J. Evaluation of lipase levels in patients with nephropathia epidemica-no evidence for acute pancreatitis. BMC Infect Dis. 2015;15:286.

2. Parsi A, Masjedizadeh A, Seyedian SS, Hashemi SJ, Jasemi F, Nourinejad R. An investigation into the sensitivity of endoscopic ultrasound in the diagnosis of malignant bile duct in patients with idiopathic acute pancreatitis. J Family Med Prim Care. 2019;8(2):733-7.

3. Jagannath S, Garg PK. Recurrent Acute Pancreatitis: Current Concepts in the Diagnosis and Management. Curr Treat Options Gastroenterol. 2018;16(4):449-65.

4. Jin DX, Lacson R, Cochon LR, et al. A Clinical Model for the Early Diagnosis of Acute Pancreatitis in the Emergency Department. Pancreas. 2018;47(7):871-9.

5. Brown TT, Prahlow JA. Postmortem Serum Amylase and Lipase Analysis in the Diagnosis of Acute Pancreatitis. Acad Forensic Pathol. 2018;8(2):311-23.

6. Liu DS, Liu L, Gan F, Wu XL, Ye G. Acute Pancreatitis Complicated by Sheehan's Syndrome: A Case Report and Literature Review. Chin Med Sci J. 2020;35(1):95-100.

7. Xiao B, Xu HB, Jiang ZQ, Zhang J, Zhang XM. Current concepts for the diagnosis of acute pancreatitis by multiparametric magnetic resonance imaging. Quant Imaging Med Surg. 2019;9(12):1973-85.

8. Jin M, Zhang HM, Chen XF, et al. [Evaluation and Early Diagnosis of Gastrointestinal Failure in Acute Pancreatitis]. Zhongguo Yi Xue Ke Xue Yuan Xue Bao. 2020;42(1):47-54.

9. Munir F, Jamshed MB, Shahid N, Muhammad SA, Ghanem NB, Qiyu Z. Current status of diagnosis and Mesenchymal stem cells therapy for acute pancreatitis. Physiol Rep. 2019;7(21):e14170.

10. Rademacher N, Schur D, Gaschen F, Kearney M, Gaschen L. Contrast-enhanced ultrasonography of the pancreas in healthy dogs and in dogs with acute pancreatitis. Vet Radiol Ultrasound. 2016;57(1):58-64.

11. Greenbaum LD. Foreword to guidelines and good clinical practice recommendations for contrast enhanced ultrasound (CEUS) in the liver-update 2012. Ultraschall Med. 2013;34(1):7.

12. Ran L, Zhao W, Zhao Y, Bu H. Value of contrast-enhanced ultrasound in differential diagnosis of solid lesions of pancreas (SLP): A systematic review and a meta-analysis. Med (Baltim). 2017;96(28):e7463.

13. Berstad AE, Brabrand $\mathrm{K}$, Horneland $\mathrm{R}$, et al. Microbubble contrast-enhanced ultrasound in the vascular evaluation after pancreas transplantation: a single-center experience. Acta Radiol. 2019;60(10):1224-31.

14. Fujita $\mathrm{M}$, Itoi $\mathrm{T}$, Ikeuchi $\mathrm{N}$, et al. Effectiveness of contrast-enhanced endoscopic ultrasound for detecting mural nodules in intraductal papillary mucinous neoplasm of the pancreas and for making therapeutic decisions. Endosc Ultrasound. 2016;5(6):377-83.

15. Huang $Z$, Tang L. [Diagnosis and treatment of peripancreatic infection in severe acute pancreatitis]. Zhonghua Wei Chang Wai Ke Za Zhi. 2018;21(12):1361-5. 
16. Ripollés T, Martínez MJ, López E, Castelló I, Delgado F. Contrast-enhanced ultrasound in the staging of acute pancreatitis. Eur Radiol. 2010;20(10):2518-23.

17. Enshaei A, Mohammadi A, Moosavi Toomatari SB, et al. Diagnostic value of a power Doppler ultrasound-based malignancy index for differentiating malignant and benign solid breast lesions. Indian J Cancer. 2020;57(1):44-8.

18. Simsek $\mathrm{O}$, Kocael A, Kocael P, et al. Inflammatory mediators in the diagnosis and treatment of acute pancreatitis: pentraxin-3, procalcitonin and myeloperoxidase. Arch Med Sci. 2018;14(2):288-96.

19. Majidi S, Golembioski A, Wilson SL, Thompson EC. Acute Pancreatitis: Etiology, Pathology, Diagnosis, and Treatment. South Med J. 2017;110(11):727-32.

20. Cho MK, Moon SH, Song TJ, et al. Contrast-Enhanced Endoscopic Ultrasound for Differentially Diagnosing Autoimmune Pancreatitis and Pancreatic Cancer. Gut Liver. 2018;12(5):591-6.

21. Rana SS, Sharma R, Guleria S, Gupta R. Endoscopic ultrasound (EUS) elastography and contrast enhanced EUS in groove pancreatitis. Indian J Gastroenterol. 2018;37(1):70-1.

22. Golea A, Badea R, Socaciu M, Diaconu B, lacob D. Quantitative analysis of tissue perfusion using contrast-enhanced transabdominal ultrasound (CEUS) in the evaluation of the severity of acute pancreatitis. Med Ultrason. 2010;12(3):198-204.

23. Wang Y, Yan K, Fan Z, et al. Clinical Value of Contrast-Enhanced Ultrasound Enhancement Patterns for Differentiating Focal Pancreatitis From Pancreatic Carcinoma: A Comparison Study With Conventional Ultrasound. J Ultrasound Med. 2018;37(3):551-9.

24. Vitali F, Pfeifer L, Janson C, et al. Quantitative perfusion analysis in pancreatic contrast enhanced ultrasound (DCE-US): a promising tool for the differentiation between autoimmune pancreatitis and pancreatic cancer. Z Gastroenterol. 2015;53(10):1175-81.

25. Cai D, Parajuly SS, Wang $\mathrm{H}$, et al. Accuracy of contrast-enhanced ultrasound compared with conventional ultrasound in acute pancreatitis: Diagnosis and complication monitoring. Exp Ther Med. 2016;12(5):3189-94.

26. Furey C, Buxbaum J, Chambliss AB. A review of biomarker utilization in the diagnosis and management of acute pancreatitis reveals amylase ordering is favored in patients requiring laparoscopic cholecystectomy. Clin Biochem. 2020;77:54-6.

27. Hocke $M$, Ignee A, Dietrich CF. Contrast-enhanced endoscopic ultrasound in the diagnosis of autoimmune pancreatitis. Endoscopy. 2011;43(2):163-5.

28. D'Onofrio M, Ciaravino V, Crosara S, De Robertis R, Pozzi Mucelli R. Contrast-Enhanced Ultrasound (CEUS) of Pancreatic Cancer. Curr Radiol Rep. 2015;3(3):6.

\section{Figures}




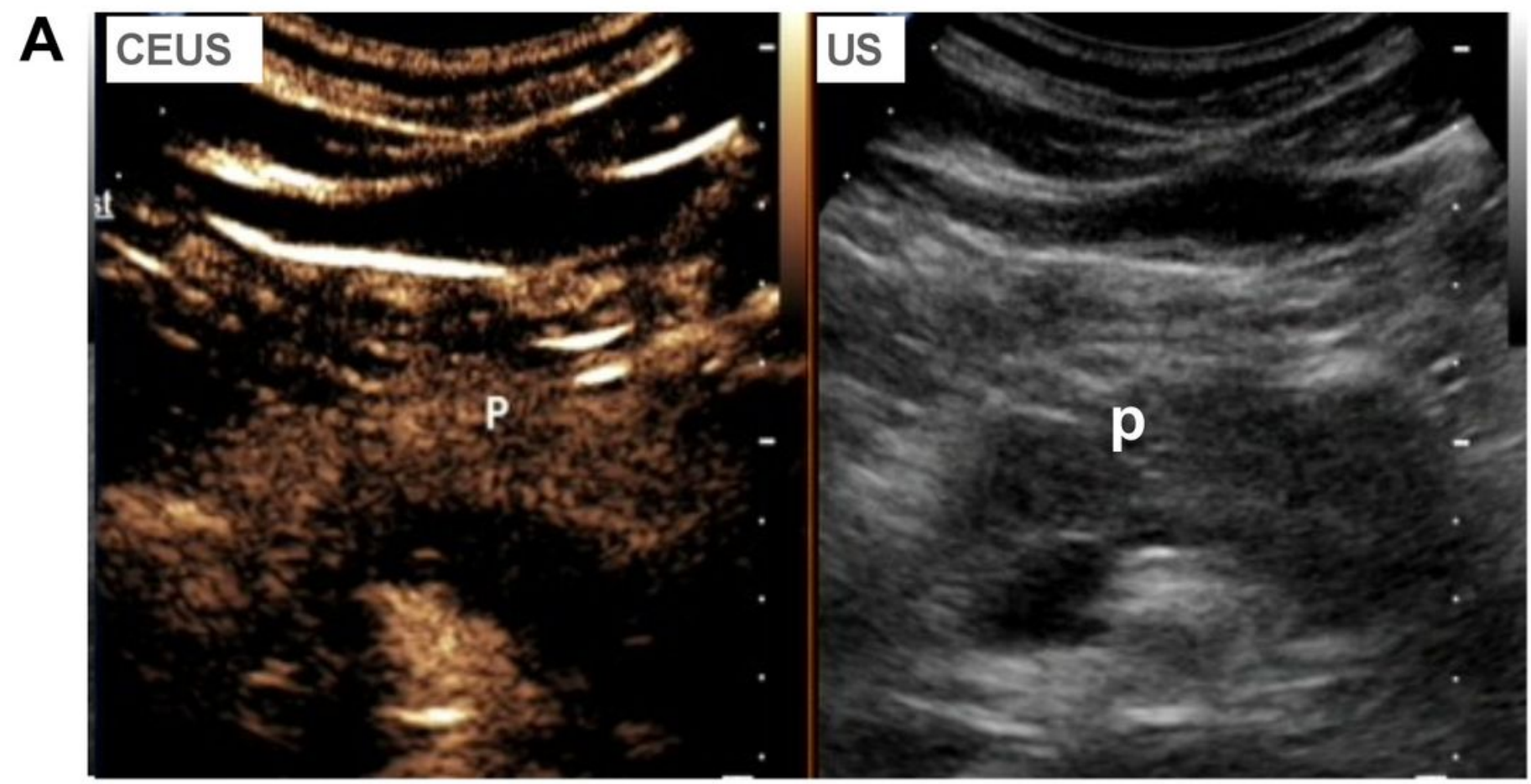

B

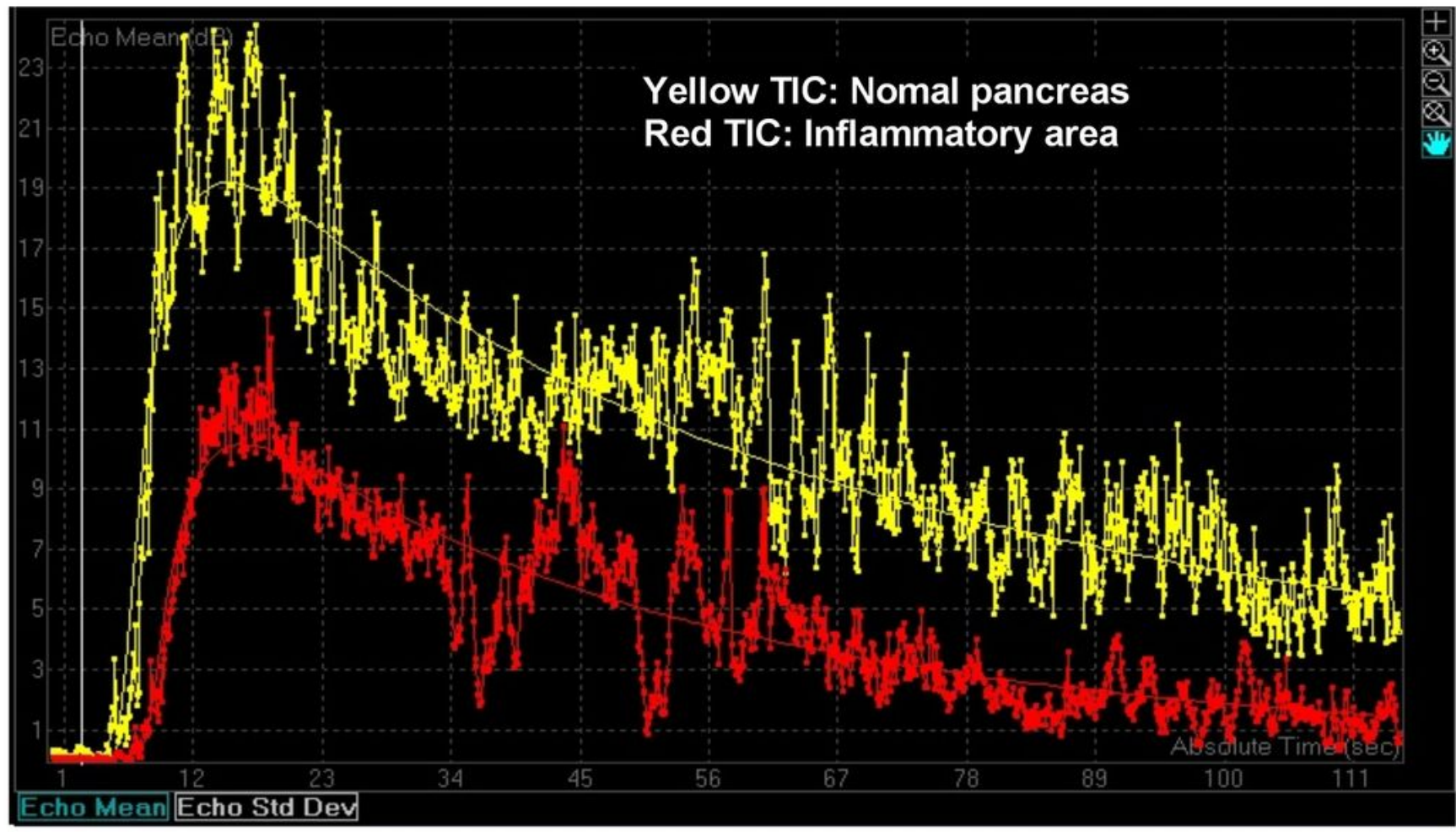

Figure 1

Ultrasound image of acute pancreatitis. A. CEUS and US image of AP B. TIC of AP and normal pancreas 


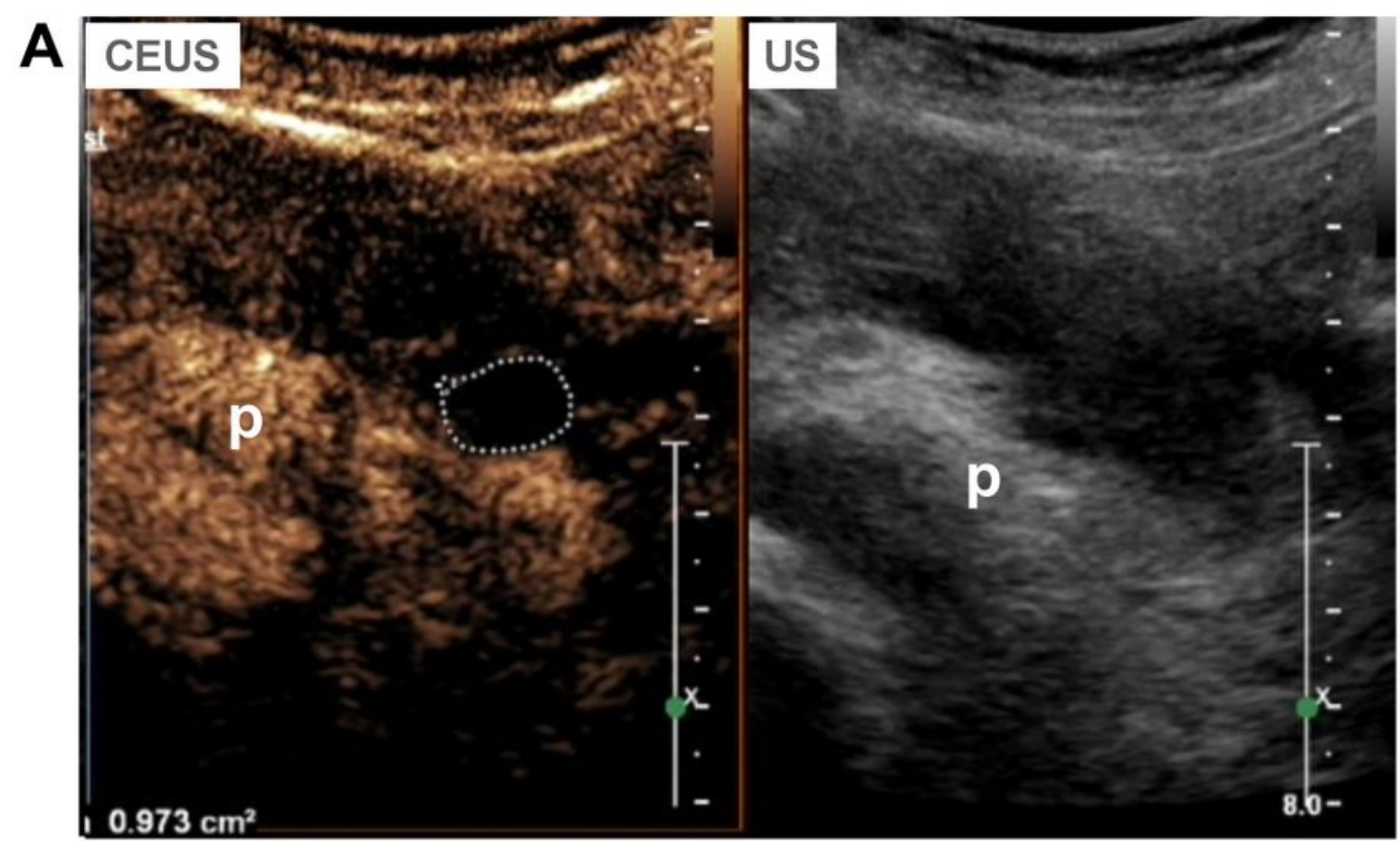

B

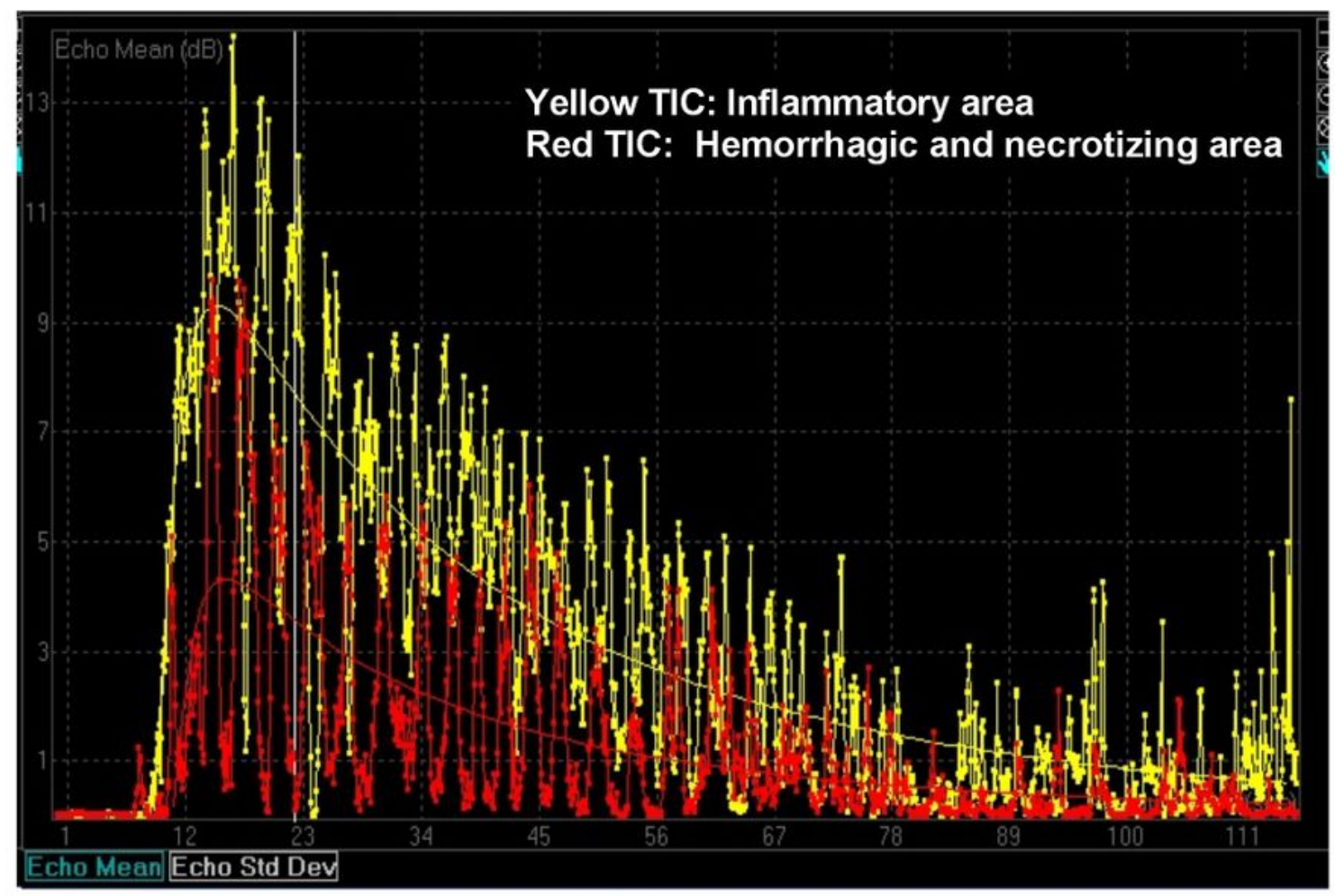

Figure 2

Ultrasound image of severe acute pancreatitis. A. CEUS and US image of SAP. B. TIC of MAP and SAP 

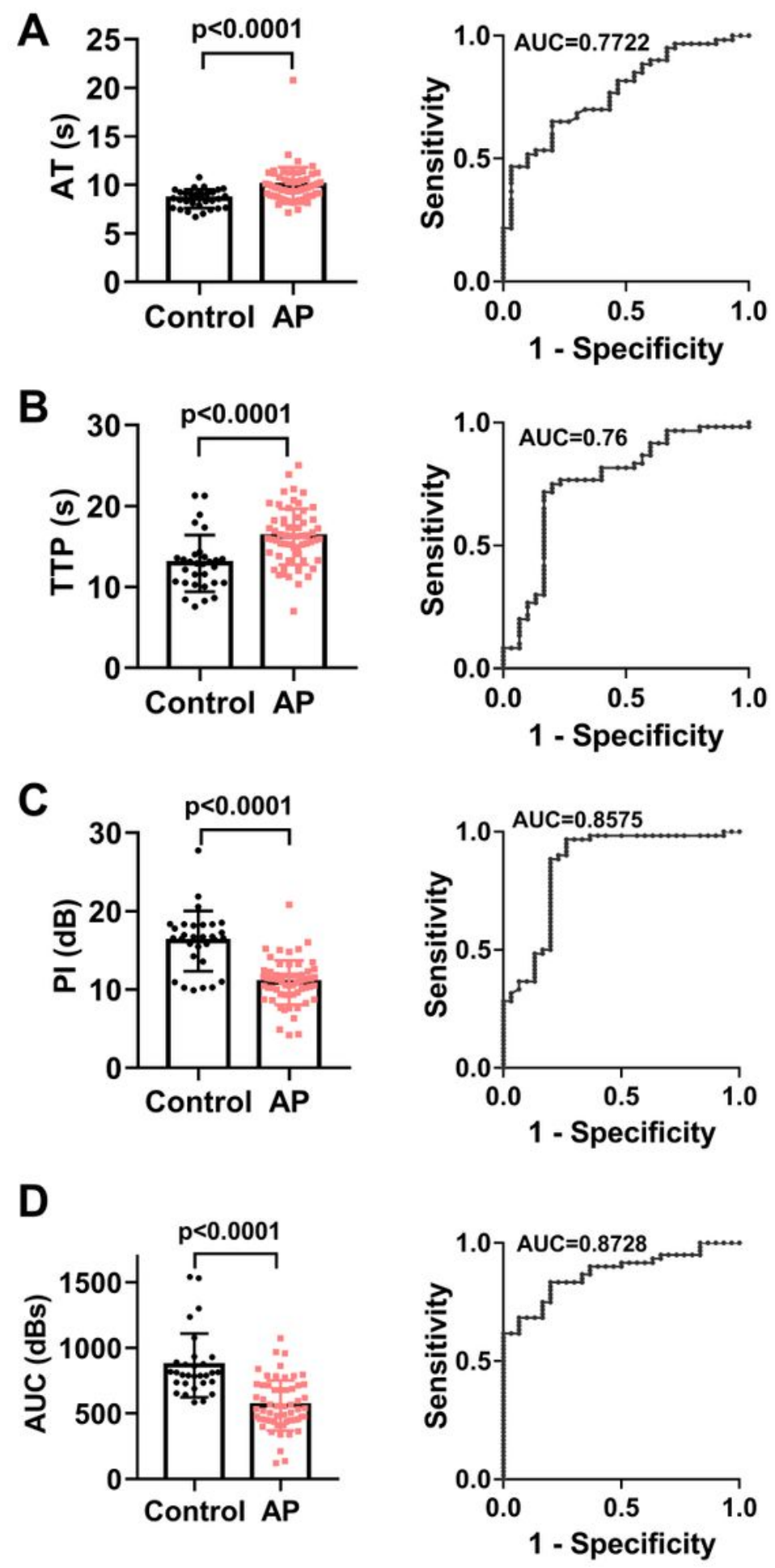

Figure 3

ROC curve comparison of different parameters. A. AT comparison between AP group and control group. B. TTP comparison between AP group and control group. C. PI comparison between AP group and control group. D. AUC comparison between AP group and control group 

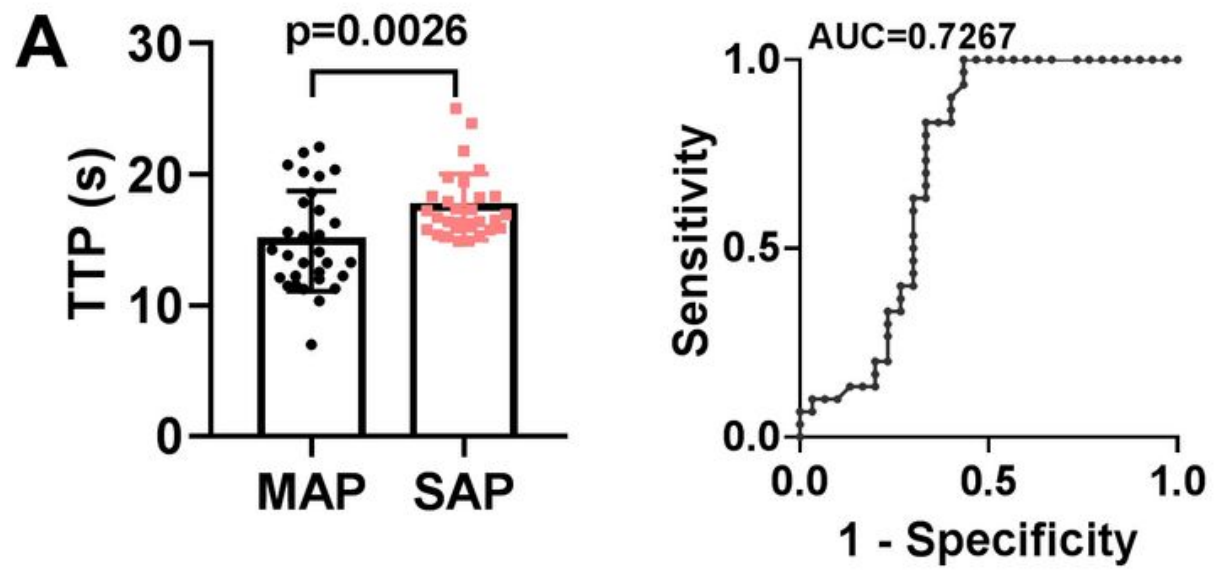

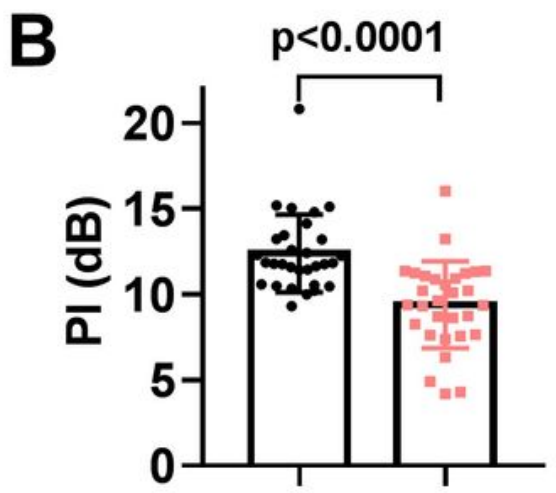

MAP SAP
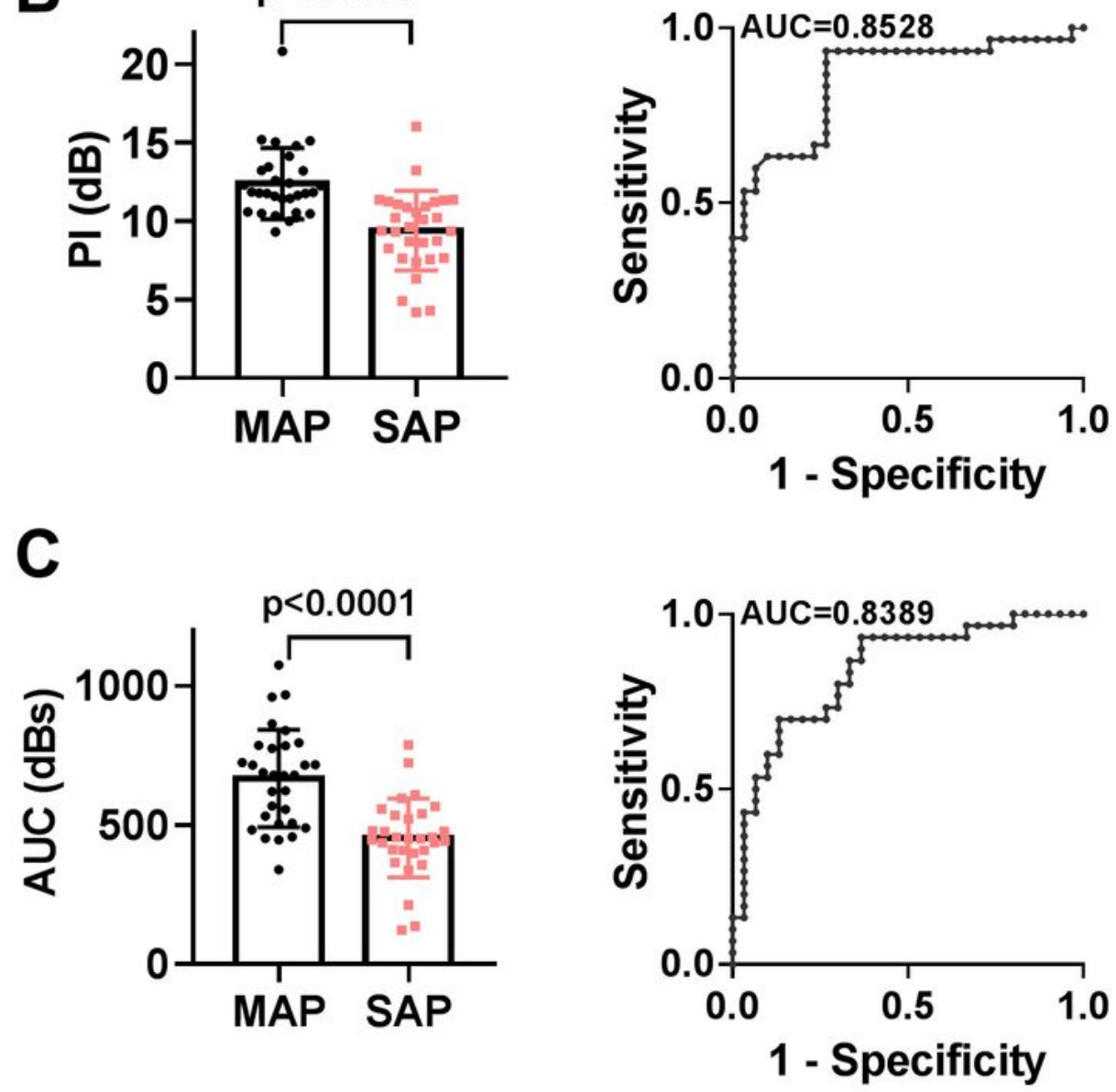

Figure 4

ROC curve comparison of different parameters. A. TTP comparison between SAP group and MAP group. B. PI comparison between SAP group and MAP group. C. AUC comparison between SAP group and MAP group 